\title{
Constructal entransy dissipation rate minimization for heat conduction based on a tapered element
}

\author{
XIAO QingHua, CHEN LinGen* \& SUN FengRui \\ College of Naval Architecture and Power, Naval University of Engineering, Wuhan 430033, China
}

Received January 23, 2011; accepted May 10, 2011

Based on constructal theory, the structure of a tapered element and high-conductivity link is optimized by taking the minimization of the entransy dissipation rate as the optimization objective. The results show that the mean temperature difference of the heat transfer cannot always decrease when the internal complexity of the control-volume increases. There exists an optimal constructal order leading to the minimum mean temperature difference for heat transfer. The thermal current density in high-conductivity links with variable shapes does not linearly depend on the length. Therefore, the optimized constructs based on the minimization of the entransy dissipation rate are different from those based on the minimization of the maximum temperature difference. Compared with the construct based on the minimization of the maximum temperature difference, the construct based on the minimization of the entransy dissipation rate can reduce the mean temperature difference, and improve the heat transfer performance significantly. Because entransy describes the heat transfer ability more suitably, various constructal problems in heat conduction may be addressed more effectively using this basis.

constructal theory, entransy dissipation rate, volume-to-point heat conduction, generalized thermodynamic optimization

Citation: Xiao Q H, Chen L G, Sun F R. Constructal entransy dissipation rate minimization for heat conduction based on a tapered element. Chinese Sci Bull, 2011, 56: 2400-2410, doi: 10.1007/s11434-011-4554-4

Many of the volume-to-point flows that occur in nature are shaped like tree networks. These flows include river basins and formative processes of cay. A volume-to-point heat conduction problem in engineering is the determination of the optimal distribution of a high-conductivity material through a finite volume, which results in the heat generated at every point being transferred most effectively to the boundary of the medium. Constructal theory was put forward by Bejan [1] and was applied to the optimization of the volume-to-point heat conduction problem. To obtain better heat transfer structure, many scholars [2-15] have made researched conduction elements with different shapes and have used various optimization objectives based on constructal theory.

Minimization of the maximum temperature difference is one of the most common optimization objectives. Bejan [1]

*Corresponding author (email: lgchenna@yahoo.com; lingenchen@hotmail.com) used the minimization of the maximum temperature difference as the optimization objective and assumed that the amount of high-conductivity material needed was finite. First, the rectangular element was optimized and the corresponding optimal elemental shape (aspect ratio) was obtained. Then, the first-order assembly that was designed with a number of optimized elemental volumes was optimized. There exists an optimal shape for the first-order assembly (or the number of the rectangular elements) that corresponds to the minimization of the maximum temperature difference of the first-order assembly. The analogy continues until the control volume is recovered by the assemblies. From [1], it can be seen that the thermal current in the high-conductivity link of a rectangular element increases continuously. Ledezma et al. [16] showed that when the constraint of a constant cross-section high-conductivity link is released and the rectangular element is re-optimized, the maximum temperature difference decreased by $6 \%$. 
Moreover, when the constraint that the high-conductivity link be perpendicular to the low-conductivity link is released, the maximum temperature difference decreases by 5.8\%. Furthermore, Almogbel and Bejan [17] proposed that when the constraint of uniform distribution of high-conductivity links through assembly was released the best highconductivity link distribution of the first-order assembly was found to be nonuniform. Also, there exist some offsets and the maximum temperature difference decreased by $5.7 \%$ when the number of the first-order assemblies was six. Ghodoossi and Egrican [18] analyzed the problem and obtained the exact results without the premise that the thermal current in the high-conductivity link of the rectangular element increases continuously, which was used in [1]. They showed that the approximate solution in [1] deviated from the obtained exact solution by $25 \%$ and that the deviation originated from the simplification that the thermal current increased continuously. Wu et al. [19] analyzed the deviation and proved that the reason was not the simplification that the thermal current increased continuously, but that equivalent coefficients of thermal conductivity were not equivalent in [1]. Wu et al. [20] re-optimized the highconductivity link distribution by releasing the constraint that the new-order assembly must be assembled using the optimized last-order assemblies and taking the aspect ratio of last-order assembly as a new optimized variable. The results showed that the maximum temperature difference of each assembly decreased by $30 \%$. Karakas et al. [21] proposed that elemental width $H_{0}$ could be substituted with $\left(D_{0}+H_{0}\right)$, and obtained optimized results which were different from those obtained in [1]. Based on a rectangular element with a variably shaped high-conductivity link, Zhou et al. [22] optimized the structure of the control-volume. The results showed that the thermal resistance cannot always decrease when the internal complexity of the control-volume increases. There exists an optimal constructal order that leads to the minimum thermal resistance. For the case in which the thermal current in the high-conductivity link increases discretely in a first- or higher-order assembly, Wei et al. [23] established a constructal optimization model with discrete variable cross-section high-conductivity links. The results showed that the minimum maximum thermal resistance of the assembly which was obtained by assembling the lastorder assemblies could be obtained by changing the cross-section of high-conductivity link at the same assembly's order. Assuming that the hot spots were distributed along the boundary uniformly and taking the minimization of maximum temperature difference as optimization objective, Neagu and Bejan [24] optimized the tapered element and high-conductivity link and obtained the optimized structure that leads to a uniform temperature field in the inner element. In addition, they showed that the minimum thermal resistance decreases by $33 \%$ compared with that obtained in [1], and by $29 \%$ compared with that when only the variable cross-section high-conductivity link was opti- mized. The thermal resistance at every point tends to be uniform in this case. Ghodoossi and Egrican [25] proposed a triangular element and obtained the optimal structure that leads to the minimum thermal resistance.

The average heat transfer effect cannot be reflected by taking the minimization of the maximum temperature difference as constructal optimization objective. Guo et al. [26] proposed a new physical quantity "entransy", which represents the global heat transfer ability. They also proposed the entransy dissipation extremum principle: For a fixed boundary heat flux, the conduction process is optimized when the entransy dissipation is minimized. However, for a fixed boundary temperature, the conduction process is optimized when the entransy dissipation is maximized. An equivalent thermal resistance for multi-dimensional heat conduction problems was defined based on the entransy dissipation. Equivalent thermal resistance is an aspect of the average heat transfer effect. The physical meaning of entransy was further explained using the physical mechanisms of heat conduction [27] and electrothermal simulation experiments [28]. Many scholars [29-37] have shown interest in heat transfer optimization based on the minimization of entransy dissipation rate. Using the minimization of entransy dissipation rate as optimization objective, Wei et al. [38-40] combined the entransy dissipation extremum principle with constructal theory for the first time and found that the construct based on the minimization of the entransy dissipation rate decreased the mean temperature difference more efficiently than the construct based on the minimization of maximum temperature difference for the same conditions. They then optimized the volume-to-point heat conduction problem with a triangular element and obtained the structure that leads to the optimal heat transfer efficiency and the equivalent thermal resistance for every assembly's order [39]. Next, they used the entransy dissipation rate minimization as optimization objective and obtained the optimal construct using the constraint that new-order assembly must be assembled by the optimized last-order assembly [40]. Finally, Wei et al. [41] derived the mean temperature difference for an electromagnet and made a multidisciplinary constructal optimization for electromagnets, which combined the magnetic density problem with the heat transfer problem. Based on the entransy dissipation rate minimization, Xie et al. $[42,43]$ optimized the heat generating bodies with rectangular cavity and T-shaped cavity. They showed that the mean temperature difference for heat transfer could be decreased more efficiently than that with maximum temperature difference minimization. This improved the global heat transfer effect in the system. The constraints that were removed from the cavity; the better the global system performance was. Chen et al. [44] optimized the disc cooling problem using the entransy dissipation rate minimization as the optimization objective.

Using a tapered element with a variable cross-section high-conductivity link, this paper will use the minimization 
of entransy dissipation rate as the constructal optimization objective. From this, we will obtain the mean temperature difference for a given control-volume and the optimal shapes of the element and high-conductivity link. We will then present some interesting conclusions via a comparison with results obtained based on the maximum temperature difference minimization in [24].

\section{Definition of entransy dissipation rate}

Entransy, which is a new physical quantity that reflects the heat transfer ability of an object, was defined in [26] as

$$
E_{v h}=\frac{1}{2} Q_{v h} T
$$

where $Q_{v h}$ is the thermal capacity of an object with a constant volume, and $T$ represents the thermal potential. The entransy dissipation function, which represents the entransy dissipation per unit time and per unit volume, was shown to be [26]

$$
\dot{E}_{h \phi}=-\dot{q} \cdot \nabla T,
$$

where $\dot{q}$ is thermal current density vector, and $\nabla T$ is the temperature gradient. During steady-state heat conduction, $\dot{E}_{h \phi}$ can be calculated as the difference between the entransy input and the entransy output of the object, i.e.

$$
\dot{E}_{h \phi}=\dot{E}_{h, \text { in }}-\dot{E}_{h, \text { out }} \text {. }
$$

The entransy dissipation rate of the whole volume in the volume-to-point conduction is

$$
\dot{E}_{v h \phi}=\int_{v} \dot{E}_{h \phi} \mathrm{d} v .
$$

The equivalent thermal resistance for multi-dimensional heat conduction problems with specified heat flux boundary conditions is given as follows [26]

$$
R_{h}=\dot{E}_{v h \phi} / \dot{Q}_{h}^{2},
$$

where $\dot{Q}_{h}$ is the thermal current. The mean temperature difference for multi-dimensional heat conduction can be expressed as

$$
\Delta \bar{T}=R_{h} \dot{Q}_{h}
$$

\section{Elemental optimization}

As shown in Figure 1 [24], the heat is generated uniformly at a rate $q^{\prime \prime \prime}\left(\mathrm{W} / \mathrm{m}^{3}\right)$ and the heat source is uniformly distributed. The heat is first directed to a high-conductivity link

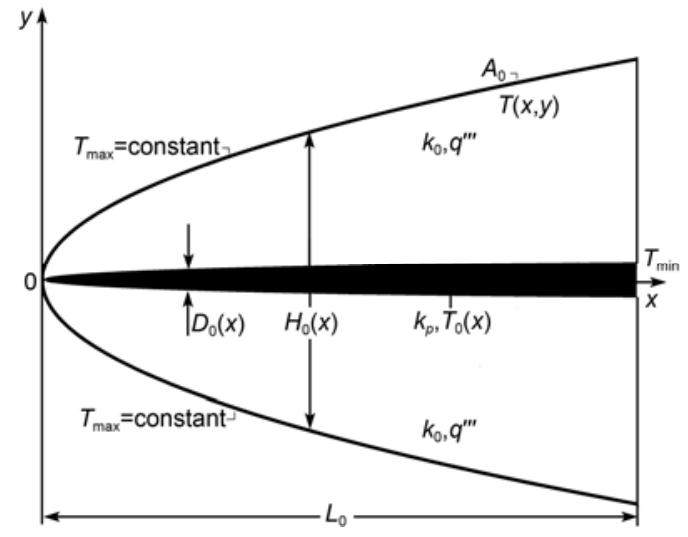

Figure 1 Tapered element [24].

(the thermal conductivity of the material is $k_{p}$, the thermal conductivity of the other material is $k_{0}$, and $k_{p} \gg k_{0}$ ) of variable width $D_{0}\left(D_{0}\right.$ is free to vary along the $x$-direction and is denoted as $\left.D_{0}(x)\right)$ and is removed from the system through a path of temperature $T_{\min }$ located at $x=L_{0}$ and $y=0\left(M_{0}\right)$. The rest of the $A_{0}$ boundary is adiabatic. The heat current flows continuously. The hot spots $\left(T_{\max }\right)$ are distributed along the boundary that does not make direct contact with $M_{0}$. This boundary is defined by the unspecified curves, $y= \pm H_{0}(x) / 2$. To allow for an analytical solution, we assume that $H_{0}(x) / L_{0} \ll 1$, and that the heat conduction in the $k_{0}$ material is along the $y$-direction and the heat conduction in the $k_{p}$ material is along the $x$-direction.

The equation [24] for heat conduction in the $k_{0}$ material is

$$
\frac{\partial^{2} T}{\partial y^{2}}+\frac{q^{\prime \prime \prime}}{k_{0}}=0
$$

with the boundary conditions

$$
\begin{gathered}
\partial T / \partial y=0, y= \pm \frac{H_{0}}{2}, \\
T=T_{0}(x), y=0,
\end{gathered}
$$

where $T_{0}(x)$ is the temperature of central $k_{p}$ blade. When $y>0$, solving eq. (7) yields

$$
T(x, y)=\frac{q^{\prime \prime \prime}}{2 k_{0}}\left(-y^{2}+H_{0} y\right)+T_{0}(x) \text {. }
$$

Setting $T=T_{\max }$ (constant) at $y= \pm H_{0} / 2$ yields

$$
T_{\max }-T_{0}(x)=\frac{q^{\prime \prime \prime} H_{0}^{2}}{8 k_{0}} .
$$

The equation for heat conduction through the $k_{p}$ blade is

$$
\frac{\mathrm{d}}{\mathrm{d} x}\left(k_{p} D_{0} \frac{\mathrm{d} T_{0}}{\mathrm{~d} x}\right)+q^{\prime \prime \prime} H_{0}=0,
$$

with boundary conditions: 


$$
\begin{gathered}
\partial T_{0} / \partial x=0, x=0, \\
T_{0}=T_{\min }, x=L_{0} .
\end{gathered}
$$

Eliminating $T_{0}(x)$ using eqs. (11) and (12) yields

$$
\frac{\mathrm{d}}{\mathrm{d} x}\left(\frac{\tilde{k} D_{0} H_{0}}{4} \frac{\mathrm{d} H_{0}}{\mathrm{~d} x}\right)=H_{0},
$$

where $\tilde{k}$ is the thermal conductivity ratio, and $\tilde{k}=k_{p} / k_{0} \gg 1$. $H_{0}$ and $D_{0}$ are the power-law functions, $H_{0}=c_{0} x^{m}, D_{0}=b_{0} x^{n}$, where $c_{0}$ and $b_{0}$ are constant factors, and $m$ and $n$ are dimensionless real numbers. From [24], one can get

$$
\begin{gathered}
\frac{\tilde{k} b_{0} c_{0} m(2 m+n-1)}{4}=1, \\
m+n=2 .
\end{gathered}
$$

And then [24]

$$
\begin{gathered}
c_{0}=\frac{2 L_{0}^{1-m}}{\left[\tilde{k} \phi_{0} m(3-m)\right]^{1 / 2}}, \\
b_{0}=\frac{2\left[\phi_{0}(3-m)\right]^{1 / 2} L_{0}^{m-1}}{(\tilde{k} m)^{1 / 2}(m+1)} .
\end{gathered}
$$

The temperature distribution is obtained by solving eq. (12):

$$
T_{0}(x)=\frac{c_{0} q^{\prime \prime \prime}}{b_{0} k_{p}(m+1)(m-n+2)}\left(L_{0}^{m-n+2}-x^{m-n+2}\right)+T_{\min } .
$$

Eliminating $T_{0}(x)$ using eqs. (10) and (20) yields the temperature difference distribution when $y>0$ :

$$
\begin{aligned}
T(x, y) & -T_{\min }=\frac{q^{\prime \prime \prime}}{2 k_{0}}\left(-y^{2}+H_{0} y\right) \\
& +\frac{c_{0} q^{\prime \prime \prime}}{b_{0} k_{p}(m+1)(m-n+2)}\left(L_{0}^{m-n+2}-x^{m-n+2}\right) .
\end{aligned}
$$

The temperature difference distribution has $x$-axis symmetry. Therefore, $H_{0}$ can be substituted with $-H_{0}$ in eq. (21) when $y<0$. The entransy dissipation rate of the element is

$$
\begin{aligned}
\dot{E}_{v h \phi 0} & =2 \int_{0}^{L_{0}} \int_{0}^{\frac{H_{0}}{2}} q^{\prime \prime \prime}\left[T(x, y)-T_{\min }\right] \mathrm{d} x \mathrm{~d} y \\
& =\frac{q^{\prime \prime \prime 2} A_{0}^{2}(m+1)(4 m+1)}{6 k_{0} \sqrt{\tilde{k} \phi_{0}}(3 m+1) \sqrt{m(3-m)}} .
\end{aligned}
$$

Setting $\mathrm{d} \dot{E}_{v h \phi 0} / \mathrm{d} m=0 \quad$ yields

$$
m_{\mathrm{opt}}=1 / 2 \text {, }
$$

Therefore, the minimum entransy dissipation rate is

$$
\dot{E}_{v h \phi 0, \mathrm{~m}}=\frac{3}{5 \sqrt{5}} A_{0}^{2} q^{\prime \prime \prime 2} k_{0}^{-1}\left(\tilde{k} \phi_{0}\right)^{-1 / 2} .
$$

The corresponding minimum equivalent thermal resistance and mean temperature difference are

$$
\begin{gathered}
R_{h 0, \mathrm{~m}}=\frac{3}{5 \sqrt{5}} k_{0}^{-1}\left(\tilde{k} \phi_{0}\right)^{-1 / 2}, \\
\Delta \bar{T}_{0}=\frac{3}{5 \sqrt{5}} A_{0} q^{\prime \prime \prime} k_{0}^{-1}\left(\tilde{k} \phi_{0}\right)^{-1 / 2} .
\end{gathered}
$$

The optimal construct of the element is:

$$
\begin{gathered}
H_{0, \text { opt }}=\frac{4}{\sqrt{5}}\left(\frac{L_{0}}{\tilde{k} \phi_{0}} x\right)^{1 / 2}, \\
D_{0, \text { opt }}=\frac{4 \sqrt{5}}{3} \sqrt{\frac{\phi_{0}}{\tilde{k} L_{0}}} x^{3 / 2}, \\
\left(\frac{H_{0, \text { base }}}{L_{0}}\right)_{\text {opt }}=\frac{4}{\sqrt{5}}\left(\tilde{k} \phi_{0}\right)^{-1 / 2} \ll 1, \\
\frac{D_{0, \text { base }}}{H_{0, \text { base }}}=\frac{5}{3} \phi_{0} .
\end{gathered}
$$

The optimal element is shown in Figure 2. Eq. (29) shows that $\tilde{k} \phi_{0} \gg 3.2$, which is in agreement with the assumed condition $H_{0}(x) / L_{0} \ll 1$. The ratio between the profile area, $A_{0}$, and the rectangle circumscribing its area, $L_{0} \times H_{0, \text { base, }}$ is $1 /(m+1)=2 / 3$. The ratio does not depend on $\tilde{k} \phi_{0}$.

Using on the minimization of the entransy dissipation rate, the optimal construct was found to be $H_{0} \propto x^{1 / 2}, D_{0} \propto x^{3 / 2}$. Moreover, the mean temperature difference and maximum temperature difference are

$$
\Delta \bar{T}_{0}=\frac{3}{5 \sqrt{5}} A_{0} q^{\prime \prime \prime} k_{0}^{-1}\left(\tilde{k} \phi_{0}\right)^{-1 / 2}=0.2683 A_{0} q^{\prime \prime \prime} k_{0}^{-1}\left(\tilde{k} \phi_{0}\right)^{-1 / 2},
$$

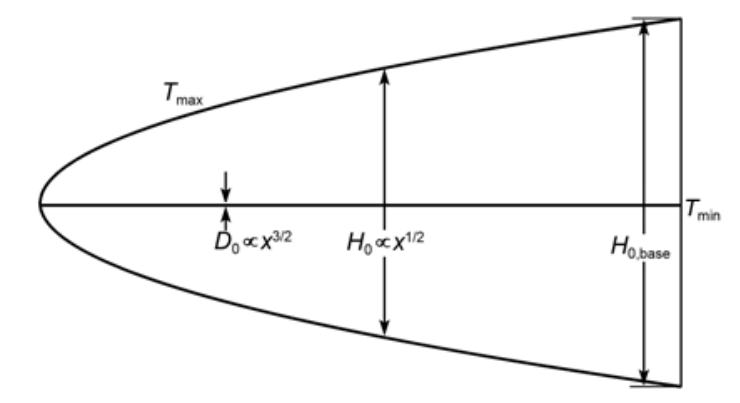

Figure 2 Optimal element. 


$$
\Delta T_{0, \max }=\frac{3}{4 \sqrt{5}} A_{0} q^{\prime \prime \prime} k_{0}^{-1}\left(\tilde{k} \phi_{0}\right)^{-1 / 2}=0.3354 A_{0} q^{\prime \prime \prime} k_{0}^{-1}\left(\tilde{k} \phi_{0}\right)^{-1 / 2} .
$$

However, using the minimization of the maximum temperature difference in [24], the optimal construct was found to be $H_{0} \propto x^{3 / 5}, D_{0} \propto x^{7 / 5}$. In this case, the mean temperature difference and maximum temperature difference are

$$
\begin{aligned}
& \Delta \bar{T}_{0}^{\prime}=\frac{17}{63} A_{0} q^{\prime \prime \prime} k_{0}^{-1}\left(\tilde{k} \phi_{0}\right)^{-1 / 2}=0.2698 A_{0} q^{\prime \prime \prime} k_{0}^{-1}\left(\tilde{k} \phi_{0}\right)^{-1 / 2}, \\
& \Delta T_{0, \text { max }}^{\prime}=\frac{1}{3} A_{0} q^{\prime \prime \prime} k_{0}^{-1}\left(\tilde{k} \phi_{0}\right)^{-1 / 2}=0.3333 A_{0} q^{\prime \prime \prime} k_{0}^{-1}\left(\tilde{k} \phi_{0}\right)^{-1 / 2} .
\end{aligned}
$$

\section{First-order assembly}

As shown in Figure 3 [24], the profile area, $A_{1}$, in the firstorder assembly is covered by a large number of optimized elements with the slendernesses indicated by eq. (29). The tips of the elements describe the unspecified curves, $y= \pm H_{1}(x) / 2$. The size of each element and its generated heat current $\left(q^{\prime \prime \prime} A_{0}\right)$ depend on the axial position, $A_{0}(x)$. The heat current is directed to the high-conductivity link, $D_{1}$, which is free to vary along the $x$-direction. It is denoted as $D_{1}(x)$. $\mathrm{Wu}$ et al. [45] showed that when using the minimization of the maximum temperature difference as optimization objective, the maximum temperatures before and after applying equivalent thermal conductivity were equal. Therefore, the entransy dissipation rates, $\dot{E}_{v h \phi 0}$ and $\dot{E}_{v h \phi 0}^{\prime}$, should be equal.

They are

$$
\begin{aligned}
\dot{E}_{v h \phi 0} & =\frac{3}{5 \sqrt{5}} A_{0}^{2} q^{\prime \prime \prime 2} k_{0}^{-1}\left(\tilde{k} \phi_{0}\right)^{-1 / 2} \\
& =\frac{64}{75 \sqrt{5}} q^{\prime \prime \prime 2} k_{0}^{-1}\left(\tilde{k} \phi_{0}\right)^{-3 / 2} L_{0}^{4},
\end{aligned}
$$

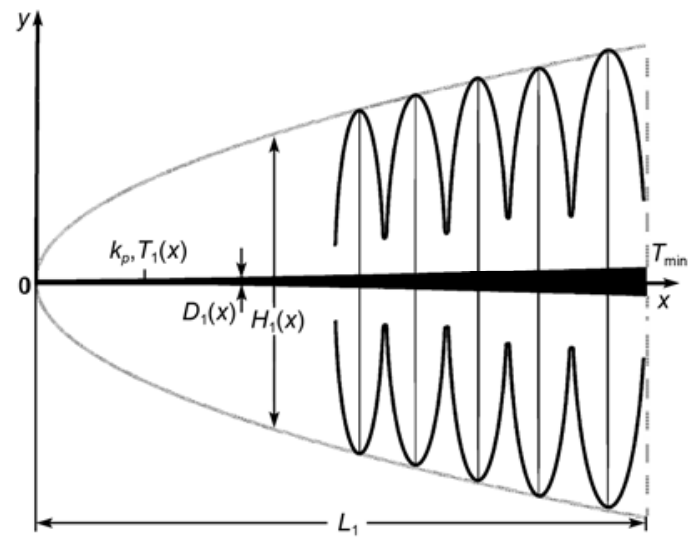

Figure 3 First-order assembly [24].

$$
\begin{aligned}
\dot{E}_{v h \phi 0}^{\prime} & =2 q^{\prime \prime \prime} \int_{0}^{L_{0}} \int_{0}^{H_{0} / 2} \frac{q^{\prime \prime \prime}}{k_{1}}\left(-\frac{x^{2}}{2}+L_{0} x\right) \mathrm{d} x \mathrm{~d} y \\
& =\frac{36}{35 \sqrt{5}} q^{\prime \prime \prime 2} k_{1}^{-1}\left(\tilde{k} \phi_{0}\right)^{-1 / 2} L_{0}^{4} .
\end{aligned}
$$

Setting them to be equal yields the equivalent thermal conductivity:

$$
k_{1}=a_{1} k_{p} \phi_{0}
$$

where $a_{1}=135 / 112$.

The equation for heat conduction through the $k_{p}$ blade is

$$
\frac{\mathrm{d}}{\mathrm{d} x}\left(k_{p} D_{1} \frac{\mathrm{d} T_{1}}{\mathrm{~d} x}\right)+\frac{2}{3} q^{\prime \prime \prime} H_{1}=0,
$$

where the factor, 2/3, accounts for the density with which the forest of elements fills the allocated area. $H_{1}$ and $D_{1}$ are the power-law functions:

$$
H_{1}=c_{1} x^{m}, D_{1}=b_{1} x^{n},
$$

where $c_{1}$ and $b_{1}$ are constant factors, and $m$ and $n$ are dimensionless real numbers. Applying eq. (15), one can obtain

$$
\frac{k_{p} b_{1} c_{1} m(2 m+n-1)}{4 k_{1}} x^{2 m+n-2}=\frac{2}{3} x^{m} .
$$

Therefore,

$$
\begin{gathered}
\frac{k_{p} b_{1} c_{1} m(2 m+n-1)}{4 k_{1}}=\frac{2}{3}, \\
m+n=2 .
\end{gathered}
$$

The profile area of the first-order assembly is

$$
A_{p, 1}=\int_{0}^{L_{1}} D_{1}(x) \mathrm{d} x+A_{1} \phi_{0}=\frac{b_{1} L_{1}^{n+1}}{n+1}+A_{1} \phi_{0} .
$$

The profile area of the $k_{p}$ blade is

$$
A_{1}=\int_{0}^{L_{1}} \frac{2}{3} H_{1}(x) \mathrm{d} x=\frac{2}{3} \frac{c_{1} L_{1}^{m+1}}{m+1} .
$$

This yields the volume fraction:

$$
\phi_{1}=\frac{A_{p, 1}}{A_{1}}=\frac{3 b_{1}(m+1) L_{1}^{n-m}}{2 c_{1}(n+1)}+\phi_{0} .
$$

Applying eq. (21), one obtains

$$
\begin{aligned}
T(x, y) & -T_{\min }=\frac{q^{\prime \prime \prime}}{2 k_{1}}\left(-y^{2}+H_{1} y\right) \\
& +\frac{2 c_{1} q^{\prime \prime \prime}}{3 b_{1} k_{p}(m+1)(m-n+2)}\left(L_{1}^{m-n+2}-x^{m-n+2}\right) .
\end{aligned}
$$


The temperature difference distribution has $x$-axis symmetry. Therefore, $H_{1}$ can be substituted with $-H_{1}$ in eq. (46) when $y<0$. The entransy dissipation rate for the first-order assembly is

$$
\begin{aligned}
\dot{E}_{v h \phi 1} & =2 \int_{0}^{L_{1}} \int_{0}^{\frac{H_{1}}{2}} \frac{2}{3} q^{\prime \prime \prime}\left[T(x, y)-T_{\min }\right] \mathrm{d} x \mathrm{~d} y \\
& =\frac{q^{\prime \prime \prime 2} A_{1}^{2}(m+1)(4 m+1)}{4 k_{p}(3 m+1) \sqrt{m(3-m)}}\left[a_{1}\left(\phi_{1}-\phi_{0}\right) \phi_{0}\right]^{-1 / 2} .
\end{aligned}
$$

By optimizing eq. (47) with respect to $m$ one obtains

$$
m_{\mathrm{opt}}=1 / 2 \text {. }
$$

The corresponding minimum entransy dissipation rate is

$$
\dot{E}_{v h \phi 1, \mathrm{~m}}=\frac{2 \sqrt{21} A_{1}^{2} q^{\prime \prime \prime 2}}{25 k_{p} \sqrt{\phi_{0}\left(\phi_{1}-\phi_{0}\right)}} .
$$

Eq. (49) shows that $\dot{E}_{v h \phi 1, \mathrm{~m}}$ decreases when either $\phi_{0}$ or $\phi_{1}$ increase. When the amount of $k_{p}$ material is fixed ( $\phi_{1}$ is a constant), optimizing eq. (49) with respect to $\phi_{0}$ yields

$$
\phi_{0, \mathrm{opt}}=\phi_{1} / 2 \text {. }
$$

The corresponding entransy dissipation rate is

$$
\dot{E}_{v h \phi 1, \mathrm{~mm}}=\frac{4 \sqrt{21} A_{1}^{2} q^{\prime \prime \prime 2}}{25 k_{p} \phi_{1}} .
$$

The equivalent thermal resistance and the mean temperature difference are

$$
\begin{gathered}
R_{h 1, \mathrm{~mm}}=\frac{4 \sqrt{21}}{25 k_{p} \phi_{1}}, \\
\Delta \bar{T}_{1}=\frac{4 \sqrt{21} A_{1} q^{\prime \prime \prime}}{25 k_{p} \phi_{1}} .
\end{gathered}
$$

The optimal construct of the first-order assembly is

$$
\begin{gathered}
H_{1, \mathrm{opt}}=\frac{3 \sqrt{21}}{7}\left(L_{1} x\right)^{1 / 2}, \\
D_{1, \mathrm{opt}}=\frac{5 \sqrt{21}}{21 \sqrt{L_{1}}} \phi_{1} x^{3 / 2}, \\
\left(\frac{H_{1, \text { base }}}{L_{1}}\right)_{\mathrm{opt}}=\frac{3 \sqrt{21}}{7}, \\
\frac{D_{1, \text { base }}}{H_{1, \text { base }}}=\frac{5}{9} \phi_{1} .
\end{gathered}
$$

The optimal first-order assembly is shown in Figure 4. Eq. (56) shows that $H_{1 \text {,base }} / L_{1}=3 \sqrt{21} / 7 \doteq 1.96>1$. Because

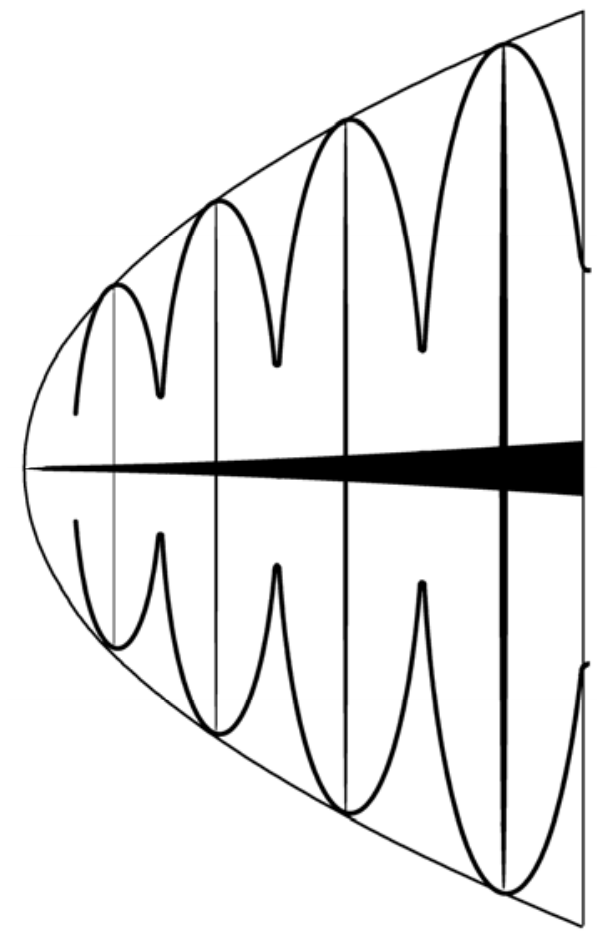

Figure 4 Optimal first-order assembly.

there is a high-conductivity link in each element, the direction of heat conduction in the $k_{1}$ area is perpendicular to the high-conductivity link, $D_{1}$. It is unnecessary to assume that $H_{1}(x) / L_{1} \ll 1$.

Based on the entransy dissipation rate minimization, the optimal construct of the first-order assembly is $H_{1} \propto x^{1 / 2}$, $D_{1} \propto x^{3 / 2}$, and the mean temperature difference and maximum temperature difference are

$$
\begin{gathered}
\Delta \bar{T}_{1}=\frac{4 \sqrt{21} A_{1} q^{\prime \prime \prime}}{25 k_{p} \phi_{1}}=0.7332 \frac{A_{1} q^{\prime \prime \prime}}{k_{p} \phi_{1}}, \\
\Delta T_{1, \max }=\frac{\sqrt{21} A_{1} q^{\prime \prime \prime}}{5 k_{p} \phi_{1}}=0.9165 \frac{A_{1} q^{\prime \prime \prime}}{k_{p} \phi_{1}} .
\end{gathered}
$$

However, using the maximum temperature difference minimization in [24], the optimal construct of the first-order assembly is $H_{1} \propto x^{3 / 5}, D_{1} \propto x^{7 / 5}$, and the mean temperature difference and maximum temperature difference are

$$
\begin{gathered}
\Delta \bar{T}_{1}^{\prime}=\frac{68 A_{1} q^{\prime \prime \prime}}{9 \sqrt{105} k_{p} \phi_{1}}=0.7373 \frac{A_{1} q^{\prime \prime \prime}}{k_{p} \phi_{1}} \\
\Delta T_{1, \max }^{\prime}=\frac{8 A_{1} q^{\prime \prime \prime}}{9 k_{p} \phi_{1}}=0.8889 \frac{A_{1} q^{\prime \prime \prime}}{k_{p} \phi_{1}} .
\end{gathered}
$$

When the element and first-order assemblies have the same applied conditions, namely, $A_{0}=A_{1}=A, \phi_{0}=\phi_{1}=\phi$, one can obtain 


$$
\Delta \bar{T}_{0} / \Delta \bar{T}_{1}=\frac{3 \sqrt{5}}{4 \sqrt{21}}(\tilde{k} \phi)^{1 / 2} .
$$

Two mean temperature difference curves between the element and first-order assemblies can be obtained. They are shown in Figure 5. When $\tilde{k} \phi>112 / 15 \approx 7.47, \Delta \bar{T}_{0} / \Delta \bar{T}_{1}>1$, namely, the mean temperature difference of the first-order design is smaller than that of the elemental design. When $\tilde{k} \phi<7.47, \Delta \bar{T}_{0} / \Delta \bar{T}_{1}<1$, namely, the mean temperature difference of the elemental design is smaller than that of the first-order design. The above section shows that $H_{0}(x) / L_{0}$ $\ll 1, \tilde{k} \phi>3.2 . \tilde{k} \phi=7.47$ is a critical value. When $3.2<\tilde{k} \phi<$ 7.47, the optimal design is the elemental one; when $\tilde{k} \phi>$ 7.47 the internal complexity should be increased, namely, the first-order design should be adopted.

\section{Second-order assembly}

As shown in Figure 6 [24], the profile area $A_{2}$ in the second-order assembly is covered by a large number of the optimized first-order assemblies. The tips of the first-order assemblies describe the unspecified curves $y= \pm H_{2}(x) / 2$. The generated heat current is directed along the high-conductivity link, $D_{2}$, which is free to vary along the $x$-direction. It is

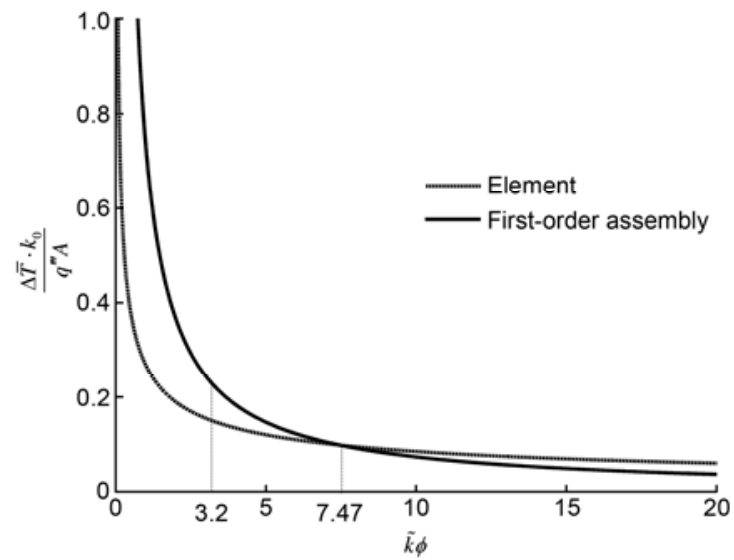

Figure 5 Mean temperature difference for the element and first-order assembly.

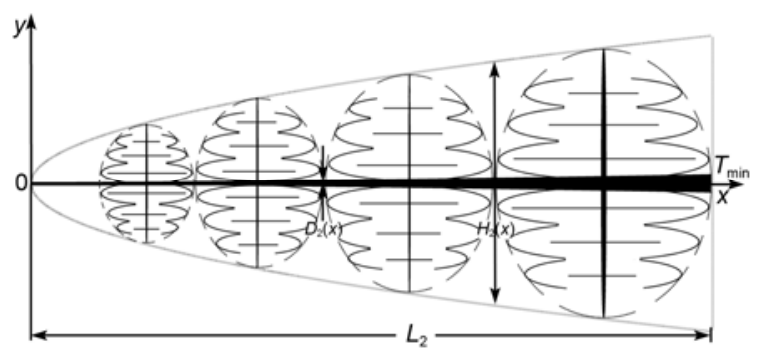

Figure 6 Second-order assembly [24]. denoted as $D_{2}(x)$. Assuming that $H_{2}(x) / L_{2} \ll 1$, the heat conduction in the $k_{2}$ material is along the $y$-direction and the heat conduction in the $k_{p}$ blade is along the $x$-direction.

Using the entransy dissipation rate minimization as the optimization objective, the entransy dissipation rates before and after applying equivalent thermal conductivity, $\dot{E}_{v h \phi 1}$ and $\dot{E}_{v h \phi 1}^{\prime}$, should be equal. They are

$$
\begin{gathered}
\dot{E}_{v h \phi 1}=\frac{4 \sqrt{21} A_{1}^{2} q^{\prime \prime \prime 2}}{25 k_{p} \phi_{1}}=\frac{64 \sqrt{21} q^{\prime \prime \prime 2} L_{1}^{4}}{525 \phi_{1} k_{p}}, \\
\dot{E}_{v h \phi 1}^{\prime}=2 q^{\prime \prime \prime} \int_{0}^{L_{1}} \int_{0}^{H_{1} / 2} \frac{2}{3} \frac{q^{\prime \prime \prime}}{k_{2}}\left(-\frac{x^{2}}{2}+L_{1} x\right) \mathrm{d} x \mathrm{~d} y \\
=\frac{18 \sqrt{21} q^{\prime \prime \prime 2} L_{1}^{4}}{245 k_{2}} .
\end{gathered}
$$

Setting them to be equal yields the equivalent thermal conductivity:

$$
k_{2}=a_{2} k_{p} \phi_{1},
$$

where $a_{2}=135 / 224$. Analogous to the above two sections, one can obtain

$$
\begin{aligned}
\dot{E}_{v h \phi 2} & =2 \int_{0}^{\frac{H_{2}}{2}} \int_{0}^{L_{2}}\left(\frac{2}{3}\right)^{2} q^{\prime \prime \prime}\left[T(x, y)-T_{\min }\right] \mathrm{d} x \mathrm{~d} y \\
& =\frac{3 q^{\prime \prime \prime 2} A_{2}^{2}(m+1)(4 m+1)}{8 k_{p}(3 m+1) \sqrt{m(3-m)}}\left[a_{2}\left(\phi_{2}-\phi_{1}\right) \phi_{1}\right]^{-1 / 2},
\end{aligned}
$$

where $\phi_{2}$ is the fixed volume fraction of the $k_{p}$ material allocated to the second-order assembly, $\phi_{2}=A_{p, 2} / A_{2} ; A_{2}$ is the profile area of the second-order assembly, $A_{2}=\int_{0}^{L_{2}}\left(\frac{2}{3}\right)^{2}$. $H_{2}(x) \mathrm{d} x ; A_{p, 2}$ is the profile area of the $k_{p}$ bladem, $A_{p, 2}=$ $\int_{0}^{L_{2}} D_{2}(x) \mathrm{d} x+A_{2} \phi_{1}$; and the factor $(2 / 3)^{2}$ accounts for the volume fraction that the heat generating material $\left(A_{2}\right)$ comprises in the second-order assembly. Optimizing eq. (66) yields

$$
\begin{gathered}
m_{\mathrm{opt}}=1 / 2, \\
\phi_{1, \mathrm{opt}}=\phi_{2} / 2 .
\end{gathered}
$$

The minimum entransy dissipation rate of the secondorder assembly is:

$$
\dot{E}_{v h \phi 2, \mathrm{~mm}}=\frac{6 \sqrt{42} A_{2}^{2} q^{\prime \prime \prime 2}}{25 k_{p} \phi_{2}} .
$$

Its corresponding minimum equivalent thermal resistance and mean temperature difference are

$$
R_{h 2, \mathrm{~mm}}=\frac{6 \sqrt{42}}{25 k_{p} \phi_{2}},
$$




$$
\Delta \bar{T}_{2}=\frac{6 \sqrt{42} A_{2} q^{\prime \prime \prime}}{25 k_{p} \phi_{2}}
$$

The optimal construct of the second-order assembly is

$$
\begin{gathered}
H_{2, \text { opt }}=\frac{3 \sqrt{42}}{14}\left(L_{2} x\right)^{1 / 2}, \\
D_{2, \text { opt }}=\frac{5 \sqrt{42}}{63 \sqrt{L_{2}}} \phi_{2} x^{3 / 2}, \\
\left(\frac{H_{2, \text { base }}}{L_{2}}\right)_{\text {opt }}=\frac{3 \sqrt{42}}{14} \approx 1.39, \\
\frac{D_{2, \text { base }}}{H_{2, \text { base }}}=\frac{10}{27} \phi_{2} .
\end{gathered}
$$

Eq. (74) shows that this construct does not agree with the assumed condition, $H_{2}(x) / L_{2} \ll 1$. It is necessary to modify the second-order assembly. The modified second-order assembly is analyzed in the following text.

As shown in Figure 7 [24], the second-order assembly can be fitted using only two optimal first-order assemblies. Its profile area is $A_{2}=2 A_{1}$. The heat currents $\left(q_{1}^{\prime}=2 q^{\prime \prime \prime} A_{1}\right)$ collected by each first-order assembly flow through the $k_{p}$ link along the $x$-direction. They are removed through the $M$ spot:

$$
q_{1}^{\prime}=k_{p} D_{2} \frac{T_{c}-T_{\min }}{H_{1, \text { base }} / 2} .
$$

The entransy dissipation rate of the $k_{p}$ link along the $x$-direction is

$$
\dot{E}_{k p}=q_{1}^{\prime}\left(T_{c}-T_{\min }\right)=\frac{2 q^{\prime 2} A_{1}^{2} H_{1, \text { base }}}{k_{p} D_{2}} .
$$

The entransy dissipation rate of the second-order assembly

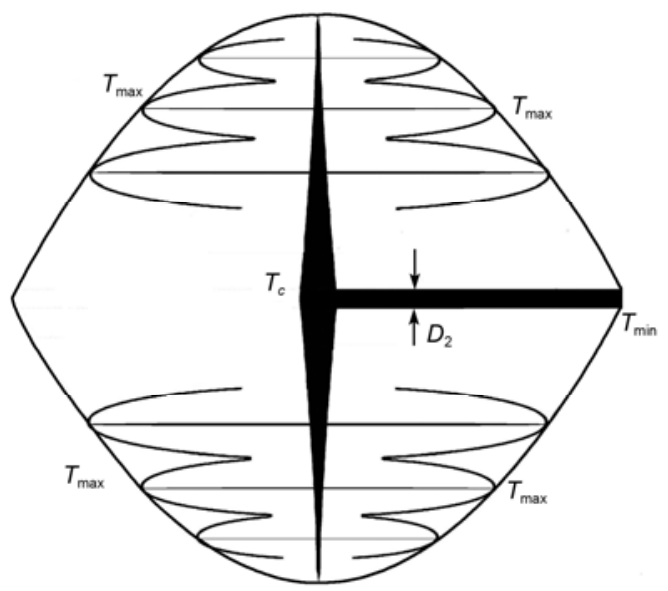

Figure 7 Modified second-order assembly. constitutes of the entransy dissipation rate of the first-order assembly and $\dot{E}_{k p}$. Namely,

$$
\dot{E}_{v h \phi 2}=\frac{2 q^{\prime \prime \prime 2} A_{1}^{2}}{k_{p}}\left(\frac{H_{1, \text { base }}}{D_{2}}+\frac{2 \sqrt{21}}{25 \phi_{1}}\right) .
$$

The volume fraction of the $k_{p}$ material allocated to the second-order assembly is

$$
\phi_{2}=\frac{A_{p, 2}}{A_{2}}=\frac{\phi_{1} A_{2}+H_{1, \text { base }} D_{2} / 2}{A_{2}}=\phi_{1}+\frac{81 D_{2}}{16 \sqrt{21} H_{1, \text { base }}} .
$$

Eliminating $H_{1 \text {,base }} / D_{2}$ between eqs. (78) and (79) and optimizing eq. (78) with respect to $\phi_{1}$ yields

$$
\begin{gathered}
\phi_{1}=0.3655 \phi_{2}, \\
\dot{E}_{v h \phi 2, \mathrm{~m}}=\frac{1.3721 q^{\prime \prime \prime 2} A_{2}^{2}}{k_{p} \phi_{2}} .
\end{gathered}
$$

The corresponding minimum equivalent thermal resistance and mean temperature difference are

$$
\begin{gathered}
R_{h 2, \mathrm{~mm}}=\frac{1.3721}{k_{p} \phi_{2}}, \\
\Delta \bar{T}_{2}=\frac{1.3721 A_{2} q^{\prime \prime \prime}}{k_{p} \phi_{2}} .
\end{gathered}
$$

Eliminating $\phi_{1}$ using eqs. (79) and (80) yields

$$
\left(\frac{D_{2}}{H_{1, \text { base }}}\right)_{\mathrm{opt}}=0.574 \phi_{2} \text {. }
$$

Based on the minimization of the entransy dissipation rate, the maximum temperature difference of the secondorder assembly is

$$
\Delta T_{2, \max }=\frac{1.8464 A_{2} q^{\prime \prime \prime}}{k_{p} \phi_{2}} .
$$

However, based on the minimization of the maximum temperature difference in [24], the mean temperature difference and the maximum temperature difference of the second-order assembly are

$$
\begin{gathered}
\Delta \bar{T}_{2}^{\prime}=\frac{1.4915 A_{2} q^{\prime \prime \prime}}{k_{p} \phi_{2}}, \\
\Delta T_{2, \max }^{\prime}=1.6877 \frac{A_{2} q^{\prime \prime \prime}}{k_{p} \phi_{2}} .
\end{gathered}
$$

When the first-order assembly and second-order assembly have the same applied conditions, namely, $A_{1}=A_{2}=A$, $\phi_{1}=\phi_{2}=\phi$, one can obtain $\Delta \bar{T}_{1} / \Delta \bar{T}_{2}=0.40<1$. Namely, the mean temperature difference of the first-order design is 
smaller than that of the second-order design.

\section{Optimization results and analysis}

If the volume fraction of high-conductivity material in each order assembly $\left(\phi_{i}(i=0,1,2)\right)$ and the profile area of each order assembly $\left(A_{i}\right)$ are kept constant, then $\phi_{0}=\phi_{1}=\phi_{2}=\phi$ and $A_{0}=A_{1}=A_{2}=A$. From above three sections, one can obtain that when $3.2<\tilde{k} \phi<7.47$, the mean temperature difference of the elemental design is smaller than that of the first-order design. Also, when $\tilde{k} \phi>7.47$, the conclusion is reversed. $\tilde{k} \phi>7.47$ is the critical point for a tree-like design. When $3.2<\tilde{k} \phi<7.47$, the optimal design for the given controlvolume is the elemental one; when $\tilde{k} \phi>7.47$ the internal complexity increases, namely, the first-order design should be adopted.

Using the minimization of the entransy dissipation rate, the constructs are optimized. The optimization results from this study are different from those obtained in [24]. The major findings of this study are listed in Table 1. The major optimization results based on the minimization of the maximum temperature difference [24] are listed in Table 2. Comparing Tables 1 and 2, it can be seen that the optimized constructs based on the minimization of the entransy dissipation rate are different from those based on the minimization of the maximum temperature difference. This is because the thermal current density in the high-conductivity links of variable shape does not vary linearly with the length [39]. From the comparison between the two tables, the optimized construct based on the maximum temperature difference minimization reduces the maximum temperature difference. However, the optimized construct based on the entransy dissipation rate minimization reduces the mean thermal resistance and improve the overall heat transfer effect.

\section{Conclusions}

By applying constructal optimization to a tapered element with a variable cross-section high-conductivity link using the minimization of entransy dissipation rate, the element is optimized. The optimal shapes of element and high-conductivity link were found to be $H_{0} \propto x^{1 / 2}, D_{0} \propto x^{3 / 2}$. These findings are different from those that were optimized $\left(H_{0} \propto x^{3 / 5}\right.$, $D_{0} \propto x^{7 / 5}$ ) using on the minimization of the maximum temperature difference. The results show that the mean temperature difference of the heat transfer cannot always decrease when the internal complexity of the control-volume increases. There is an optimal constructal order that leads to the minimum mean temperature difference for heat transfer. When $3.2<\tilde{k} \phi<7.47$, the optimal design for the given controlvolume is the elemental one. Namely, the optimal constructal order, which is zero, leads to the minimum mean temperature difference for heat transfer in the control-volume. When $\tilde{k} \phi>7.47$, the internal complexity is increased and the firstorder design should be adopted. Namely, the optimal constructal order, which is one, leads to the minimum mean temperature difference for heat transfer in the control-volume. The equivalent thermal resistance defined based on the

Table 1 Constructal optimization results based on the minimization of the entransy dissipation rate (this study)

\begin{tabular}{|c|c|c|c|c|c|}
\hline $\begin{array}{l}\text { Order of the } \\
\text { assembly, } i\end{array}$ & $\left(\frac{D_{i, \text { base }}}{H_{i, \text { base }}}\right)_{\mathrm{opt}}$ & $\left(\frac{H_{i, \text { base }}}{L_{i}}\right)_{\mathrm{opt}}$ & $\left(\frac{D_{i}}{H_{i-1, \text { base }}}\right)_{\mathrm{opt}}$ & $\frac{\Delta T_{i, \max } \cdot k_{p} \phi_{i}}{q^{\prime \prime \prime} A_{i}}$ & $\frac{\Delta \bar{T}_{i} \cdot k_{p} \phi_{i}}{q^{\prime \prime \prime} A_{i}}$ \\
\hline 0 & $\frac{5}{3} \phi_{0}$ & $\frac{4}{\sqrt{5}}\left(\tilde{k} \phi_{0}\right)^{-1 / 2}$ & - & $0.3354\left(\tilde{k} \phi_{0}\right)^{1 / 2}$ & $0.2683\left(\tilde{k} \phi_{0}\right)^{1 / 2}$ \\
\hline 1 & $\frac{5}{9} \phi_{1}$ & $\frac{3 \sqrt{21}}{7}$ & - & 0.9165 & 0.7332 \\
\hline 2 & - & - & $0.574 \phi_{2}$ & 1.8464 & 1.3721 \\
\hline
\end{tabular}

Table 2 Constructal optimization results based on the minimization of the maximum temperature difference [24]

\begin{tabular}{|c|c|c|c|c|c|}
\hline $\begin{array}{l}\text { Order of the } \\
\text { assembly, } i\end{array}$ & $\left(\frac{D_{i, \text { base }}}{H_{i, \text { base }}}\right)_{\mathrm{opt}}^{\text {a) }}$ & $\left(\frac{H_{i, \text { base }}}{L_{i}}\right)_{\mathrm{opt}}$ & $\left(\frac{D_{i}}{H_{i-1, \text { base }}}\right)_{\text {opt }}$ & $\frac{\Delta T_{i, \max } \cdot k_{p} \phi_{i}}{q^{\prime \prime \prime} A_{i}}$ & $\frac{\Delta \bar{T}_{i} \cdot k_{p} \phi_{i}^{\text {a) }}}{q^{\prime \prime \prime} A_{i}}$ \\
\hline 0 & $\frac{3}{2} \phi_{0}$ & $\frac{5}{3}\left(\tilde{k} \phi_{0}\right)^{-1 / 2}$ & - & $0.3333\left(\tilde{k} \phi_{0}\right)^{1 / 2}$ & $0.2698\left(\tilde{k} \phi_{0}\right)^{1 / 2}$ \\
\hline 1 & $\frac{15}{32} \phi_{1}$ & 2 & - & 0.8889 & 0.7373 \\
\hline
\end{tabular}

a) These columns were derived from data in [24]. 
entransy dissipation rate reflects the heat conduction ability and heat transfer efficiency in the heat transfer process. The smaller the equivalent thermal resistance; the better is the heat transfer effect and the lower the mean temperature in the control-volume. The minimum thermal resistance reflects the maximum temperature difference, and represents the maximum temperature limit for the volume. The construct based on the minimization of the entransy dissipation rate is the optimal result for the mean heat transfer effect, which differs from the construct based on the minimization of the maximum temperature difference. Compared with the optimal constr uct based on the minimization of the maximum temperature difference, the optimal construct based on the minimization of the entransy dissipation rate decreases the mean temperature difference for heat transfer significantly. The improvement in the heat transfer effect resulting from the optimal construct based on the minimization of the entransy dissipation rate can be clearly seen in our results. Both the mean temperature difference and the maximum temperature difference should be combined when considering the efficiency and temperature limits simultaneously. This is important for addressing volume-to-point heat conduction problems. This paper has fully described the effect of the minimization of the entransy dissipation rate. Because the idea of entransy describes heat transfer ability more suitably [26] than the minimization of the maximum temperature difference, we suggest that all future studies of heat conduction constructal problems be based on entransy.

This work was supported by the National Natural Science Foundation of China (10905093), the Program for New Century Excellent Talents in Universities of China (NCET-04-1006) and the Foundation for the Author of National Excellent Doctoral Dissertations of China (200136). The authors wish to thank the reviewers for their careful, unbiased and constructive suggestions, which led to this revised manuscript.

1 Bejan A. Constructal-theory network of conducting paths for cooling a heat generating volume. Trans ASME, J Heat Transfer, 1997, 40: 799-816

2 Bejan A. Shape and Structure, from Engineering to Nature. Cambridge: Cambridge University Press, 2000

3 Bejan A, Lorente S. Thermodynamic optimization of flow geometry in mechanical and civil engineering. J Non-Equilib Thermodyn, 2001, 26: 305-354

4 Zhou S, Chen L, Sun F. Constructal theory: A new direction for generalized thermodynamic optimization (in Chinese). Thermal Sci Tech, 2004, 3: 283-292

5 Rosa R, Reis A, Miguel A. Proceedings of the Symposium Bejan's Constructal Theory of Shape and Structure. Evora: University of Evora, 2004. 219

6 Bejan A, Lorente S. The Constructal Law (La Loi Constructale). Paris: L'Harmatan, 2005

7 Bejan A, Lorente S. Constructal multi-scale and multi-objective structures. Int J Energy Res, 2005, 29: 689-710

8 Bejan A, Lorente S, Miguel A, et al. Along with Constructal Theory. UNIL FGSE Workshop Series No. 1. Hernandez J, Cosinschi M, eds. University of Lausanne, Faculty of Geosciences and the Environment Switzerland, 2006

9 da Silva A, Lorente S, Bejan A. Constructal multi-scale structures for maximal heat transfer density. Energy, The Int J, 2006, 31: 620-635
10 Reis A. Constructal theory: From engineering to physics, and how flow systems develop shape and structure. Appl Mech Rev, 2006, 59: 269-282

11 Bejan A, Lorente S. Constructal theory of generation of configuration in nature and engineering. J Appl Phys, 2006, 100: 041301

12 Bejan A, Marden J H. Unifying constructal theory for scale effects in running, swimming and flying. J Experimental Biology, 2006, 209: 238-248

13 Bejan A, Merkx G, eds. Constructal Theory of Social Dynamics. New York: Springer, 2007

14 Bejan A, Lorente S. Design with Constructal Theory. New Jersey: Wiley, 2008

15 Bejan A, Lorente S, Miguel A, et al. Constructal Human Dynamics, Security \& Sustainability. Amsterdam: IOS Press, 2009

16 Ledezma G, Bejan A, Errera M. Constructal tree networks for heat transfer. J Appl Phys, 1997, 82: 89-100

17 Almogbel M, Bejan A. Constructal optimization of nonuniformly distributed tree-shaped flow structures for conduction. Int $\mathrm{J}$ Heat Mass Transfer, 2001, 44: 4185-4194

18 Ghodoossi L, Egrican N. Exact solution for cooling of electronics using constructal theory. J Appl Phys, 2003, 93: 4922-4929

$19 \mathrm{Wu} \mathrm{W}$, Chen L, Sun F. On the "area to point" flow problem based on constructal theory. Energy Convers Mgmt, 2007, 48: 101-105

$20 \mathrm{Wu}$ W, Chen L, Sun F. Improvement of tree-like network constructal method for heat conduction optimization. Sci China Ser E-Tech Sci, 2006, 49: 332-341

21 Karakas A, Camdali U, Tunc M. Constructal optimization of heat generating volumes. Int J Exergy, 2009, 6: 637-654

22 Zhou S, Chen L, Sun F. Optimization of constructal volume-point conduction with variable cross-section conducting path. Energy Convers Mgmt, 2007, 48: 106-111

23 Wei S, Chen L, Sun F. The volume-point constructal optimization for discrete variable cross-section conducting path. Appl Energy, 2009, 86: $1111-1118$

24 Neagu M, Bejan A. Constructal-theory tree networks of "constant" thermal resistance. J Appl Phys, 1999, 86: 1136-1144

25 Ghodoossi S, Egrican N. Conductive cooling of triangular shaped electronics using constructal theory. Energy Convers Mgmt, 2004, 45: 811-828

26 Guo Z, Zhu H, Liang X. Entransy-A physical quantity describing heat transfer ability. Int J Heat Mass Transfer, 2007, 50: 2545-2556

27 Han G, Guo Z. Physical mechanism of heat conduction ability dissipation and its analytical expression (in Chinese). In: Proc CSEE, 2007, 27: 98-102

28 Zhu H, Chen J, Guo Z. Electricity and thermal analogous experimental study for entransy dissipation extreme principle (in Chinese). Prog Nat Sci, 2007, 17: 1692-1698

29 Cheng X, Meng J, Guo Z. Potential capacity dissipation minimization and entropy generation minimization in heat conduction optimization (in Chinese). J Eng Thermophys, 2005, 26: 1034-1036

30 Chen Q, Ren J. Generalized thermal resistance for convective heat transfer and its relation to entransy dissipation. Chinese Sci Bull, 2008, 53: 3753-3761

31 Liu X, Guo Z, Meng J. Analyses for entransy dissipation and heat resistance in heat exchangers (in Chinese). Prog Natural Sci, 2008, 18: 1186-1190

32 Song W, Meng J, Liang X, et al. Probative uniformity principle of temperature difference field in one-dimensional heat exchanger (in Chinese). J Chem Eng, 2008, 59: 2460-2464

33 Liu X, Meng J, Guo Z. Entropy generation extremum and entransy dissipation extremum for heat exchanger optimization. Chinese Sci Bull, 2009, 54: 943-947

$34 \mathrm{Wu}$ J, Liang X. Application of entransy dissipation extremum principle in radiative heat transfer optimization. Sci China Ser E-Tech Sci, 2008, 51: 1306-1314

35 Xia S, Chen L, Sun F. Optimization for entransy dissipation minimization in heat exchanger. Chinese Sci Bull, 2009, 54: 3587-3595

36 Wang S, Chen Q, Zhang B. An equation of entransy and its application. Chinese Sci Bull, 2009, 54: 3572-3578 
37 Chen Q, Wang M, Pan N, et al. Optimization principles for convective heat transfer. Energy, 2009, 34: 1199-1206

38 Wei S, Chen L, Sun F. "Volume-point" heat conduction constructal optimization with entransy dissipation minimization objective based on rectangular element. Sci China Ser E-Tech Sci, 2008, 51: 1283-1295

39 Wei S, Chen L, Sun F. Constructal entransy dissipation minimization for "volume-point" heat conduction based on triangular element. Therm Sci, 2010, 14: 1075-1088

40 Wei S, Chen L, Sun F. Constructal entransy dissipation minimization for "volume-point" heat conduction without the premise of optimized last-order construct. Int J Exergy, 2010, 7: 627-639

41 Wei S, Chen L, Sun F. Constructal multidisciplinary optimization of electromagnet based on entransy dissipation minimization. Sci China Ser E-Tech Sci, 2009, 52: 2981-2989

42 Xie Z, Chen L, Sun F. Constructal optimization on T-shaped cavity based on entransy dissipation minimization. Chinese Sci Bull, 2009, 54: 4418-4427

43 Xie Z, Chen L, Sun F. Constructal optimization for geometry of cavity by taking entransy dissipation minimization as objective. Sci China Ser E-Tech Sci, 2009, 52: 3504-3513

44 Chen L, Wei S, Sun F. Constructal entransy dissipation rate minimization of a disc. Int J Heat Mass Transfer, 2011, 54: 210-216

$45 \mathrm{Wu}$ W, Chen L, Sun F. On the "area to point" flow problem based on constructal theory. Energy Convers Mgmt, 2007, 48: 101-105

Open Access This article is distributed under the terms of the Creative Commons Attribution License which permits any use, distribution, and reproduction in any medium, provided the original author(s) and source are credited. 